\title{
Autoinforme de salud general en adultos mayores de América Latina y el Caribe: su utilidad como indicador
}

\author{
Rebeca Wong, ${ }^{1}$ Martha Peláez ${ }^{2}$ y Alberto Palloni ${ }^{3}$
}

Forma de citar

Wong R, Peláez M, Palloni A. Autoinforme de salud general en adultos mayores de América Latina y el Caribe: su utilidad como indicador. Rev Panam Salud Publica. 2005:17(5/6):323-32.

RESUMEN Objetivos. Evaluar el autoinforme de salud general (ASG) como indicador de salud y analizar sus covariables en personas de 60 años de edad o más que residian en hogares privados en siete centros urbanos de América Latina y el Caribe.

Métodos. Estudio descriptivo transversal basado en los datos de la encuesta Salud, Bienestar y Envejecimiento (SABE) realizada entre 1999 y 2000 en: Bridgetown, Barbados; Buenos Aires, Argentina; México, D.F., México; Ciudad de La Habana, Cuba; Montevideo, Uruguay; Santiago, Chile; y São Paulo, Brasil. Se exploraron las características demográficas y socioeconómicas de los participantes, algunos indicadores de salud (autoinforme de enfermedades crónicas, padecimiento de depresión y elementos cognoscitivos), la red social y familiar de apoyo, el uso de los servicios de salud, la funcionalidad informada y observada, los ingresos del encuestado y los bienes de consumo duraderos en su vivienda. En los modelos de regresión (probit) se utilizó una salud regular o mala según el autoinforme como variable dependiente. Se empleó el efecto marginal de cada variable explicativa categórica para indicar la diferencia entre la probabilidad de informar mala salud de las personas que tenían y de las que no tenían una característica dada.

Resultados. En todas las ciudades estudiadas fue muy baja la propensión a informar salud "excelente" (6\% o menos). Los resultados del análisis con múltiples variables de la relación entre el ASG mala o regular y las covariables mostraron: 1) la importancia relativa de varios indicadores de salud como covariables del ASG; 2) la asociación entre las características sociodemográficas y el ASG; y 3) las diferencias o similitudes encontradas entre los siete centros urbanos con respecto a las relaciones estudiadas. La proporción de autoinforme de buena salud fue mayor en Buenos Aires y Montevideo (60\%), seguidas de Bridgetown y São Paulo (alrededor de 50\%) y de Ciudad de La Habana, Santiago y México, D.F. (entre 30 y 40\%). La evaluación de la propia memoria fue el factor más fuertemente asociado con el resultado del ASG, seguido de la satisfacción con el estado nutricional y con la vida.

Conclusiones. El ASG captó múltiples facetas de la salud de los adultos mayores, como el padecimiento de enfermedades crónicas, su grado de satisfacción con el nivel de nutrición y con la vida, su percepción del estado de la propia memoria y los problemas de funcionalidad que sufrían. Se deben emprender estudios más detallados que permitan establecer el papel que desempeña la salud emocional en la demanda de atención sanitaria de los adultos mayores en

Maryland Population Research Center, Universidad de Maryland, College Park, Maryland, Estados Unidos de América. La correspondencia debe dirigirse a Rebeca Wong, Maryland Population
Research Center, University of Maryland, 1103 Art-Sociology Building, College Park, Maryland 20742, EUA. Correo electrónico: rwong@pop center.umd.edu
2 Organización Panamericana de la Salud, Washington, D.C., EUA.

3 Center for Demography and Ecology, University of Wisconsin, Madison, Wisconsin, EUA. 
América Latina y el Caribe y determinar si existe alguna asociación entre el ASG y el uso de los servicios de salud.

Palabras clave Ancianos, ancianos de 80 años y más, actividades cotidianas, estado de salud.

Numerosos estudios sobre la salud de la población han utilizado el autoinforme de salud general (ASG), especialmente en adultos mayores. Su uso generalizado se debe principalmente a tres razones (1-3): primero, porque de forma agregada es un indicador asociado significativamente con la mortalidad y con la salud de la población, por lo que puede usarse con suficiente aproximación para medir su demanda de atención de salud; segundo, porque a nivel individual el ASG predice la mortalidad en personas de edad avanzada, por lo que puede emplearse en modelos de comportamiento actual o futuro para predecir, por ejemplo, la utilización de servicios o de planes de retiro; tercero, porque es un indicador relativamente fácil de aplicar mediante encuestas de una sola pregunta: "¿Cómo considera su salud general: excelente, muy buena, buena, regular o mala?" Esto implica que no se requiere de personal especializado para evaluar el estado general de la salud en la población.

Por otro lado, se ha encontrado una fuerte asociación entre los resultados del ASG y el nivel sociodemográfico de la población, pues el estado general de salud es mejor cuando el nivel socioeconómico es mayor. Sin embargo, es posible que las excepciones de esta regla en poblaciones de adultos mayores ayuden a ampliar el conocimiento acerca del proceso de envejecimiento saludable (4-6). Para investigar estas hipótesis en la población adulta mayor de América Latina y el Caribe es necesario contar con datos precisos sobre la salud de las personas y sus covariables económicas y sociales. Resulta difícil y costoso medir algunos indicadores de salud y de morbilidad en una muestra nacional, por lo que cobra importancia analizar lo que un indicador como el ASG puede ofrecer para entender la relación entre la salud y el bienestar económico y social en este grupo de población.
Además, aunque se reconoce la subjetividad del ASG debido a que depende de la percepción que las personas tienen de su propia salud, este indicador puede tener ventajas, especialmente en casos en que la población no tiene acceso generalizado a los servicios de salud (7). En los países desarrollados, donde en general existe mayor contacto entre la población y el personal sanitario, es posible que el autoinforme de enfermedades específicas - como la diabetes, la hipertensión arterial o el cáncer- sea un mejor indicador de la salud de la población, por ser más objetivo que la evaluación general de la propia salud (8). Sin embargo, aun en los países desarrollados, el autoinforme de enfermedades específicas puede encerrar grandes sesgos (9).

Gran parte de la información acerca del empleo del ASG en adultos mayores y de su relación con otros indicadores proviene de países desarrollados. La oportunidad de evaluar la utilidad de este indicador en países en desarrollo surgió recientemente con las primeras investigaciones realizadas en algunos países de América Latina y Asia $(10,11)$.

El objetivo del presente artículo es evaluar el ASG como indicador de salud y analizar el comportamiento de sus covariables en personas de 60 años de edad o más que residían en hogares privados en siete centros urbanos de América Latina y el Caribe.

\section{MATERIALES Y MÉTODOS}

Para el presente estudio descriptivo transversal se utilizaron los datos recolectados mediante la encuesta Salud, Bienestar y Envejecimiento (SABE), realizada entre 1999 y 2000 en siete centros urbanos de América Latina y el Caribe: Bridgetown, Barbados; Buenos Aires, Argentina; México, D.F., México; Ciudad de La Habana, Cuba; Montevideo,
Uruguay; Santiago, Chile; y São Paulo, Brasil. ${ }^{4}$ En total se aplicó la encuesta a 10597 personas de 60 años de edad o más que residían en sus hogares. Las personas encuestadas se seleccionaron mediante un muestreo estratificado y multietápico realizado en cada centro urbano estudiado (12). La encuesta exploró las características demográficas de los participantes, así como diversos indicadores de salud (el autoinforme de enfermedades crónicas, el padecimiento de depresión y algunos elementos cognoscitivos, entre otras), la red social y familiar de apoyo, el uso de los servicios de salud, la funcionalidad informada y observada, los ingresos y pensiones del encuestado y los bienes de consumo duraderos presentes en su vivienda. Debido a que el objetivo del trabajo era conocer la evaluación personal acerca de la propia salud y de otros elementos del bienestar personal, para este trabajo no se tomaron en cuenta los datos obtenidos a través de un informante sustituto. ${ }^{5}$

Para evitar el sesgo debido a la composición por edad y sexo de la población de cada ciudad, los datos que se presentan agregados se ponderaron y estandardizaron según la edad, empleando como estándar la distribución de edades de México, D.F. De esta manera, las diferencias observadas entre las ciudades estudiadas no dependieron de la composición por edad y sexo de sus poblaciones.

A fin de aprovechar las posibilidades que brinda el ASG para reflejar múltiples facetas de la calidad de la vida de una persona (13-17), se anali-

\footnotetext{
4 La encuesta se realizó en colaboración con centros de investigación y universidades de cada una de las ciudades seleccionadas. Los investigadores principales fueron: M. Peláez, C. Albala, J.C. Alfonso, R. Ham-Chande, A. Hennis, M.L. Lebrão, E. León-Díaz y A. Pantelides.

5 Se excluyeron 674 casos en que la entrevistas se realizó a informantes sustitutos (Buenos Aires: 40; Bridgetown: 37; São Paulo: 249; Santiago: 102; Ciudad de La Habana: 194; y México, D.F.: 52).
} 
CUADRO 1. Distribución porcentual de los autoinformes de salud, bienestar y funcionalidad por sexo y ciudad. Datos ponderados y estandardizados por la población de México, D.F. Encuesta Salud, Bienestar y Envejecimiento (SABE), 1999-2000

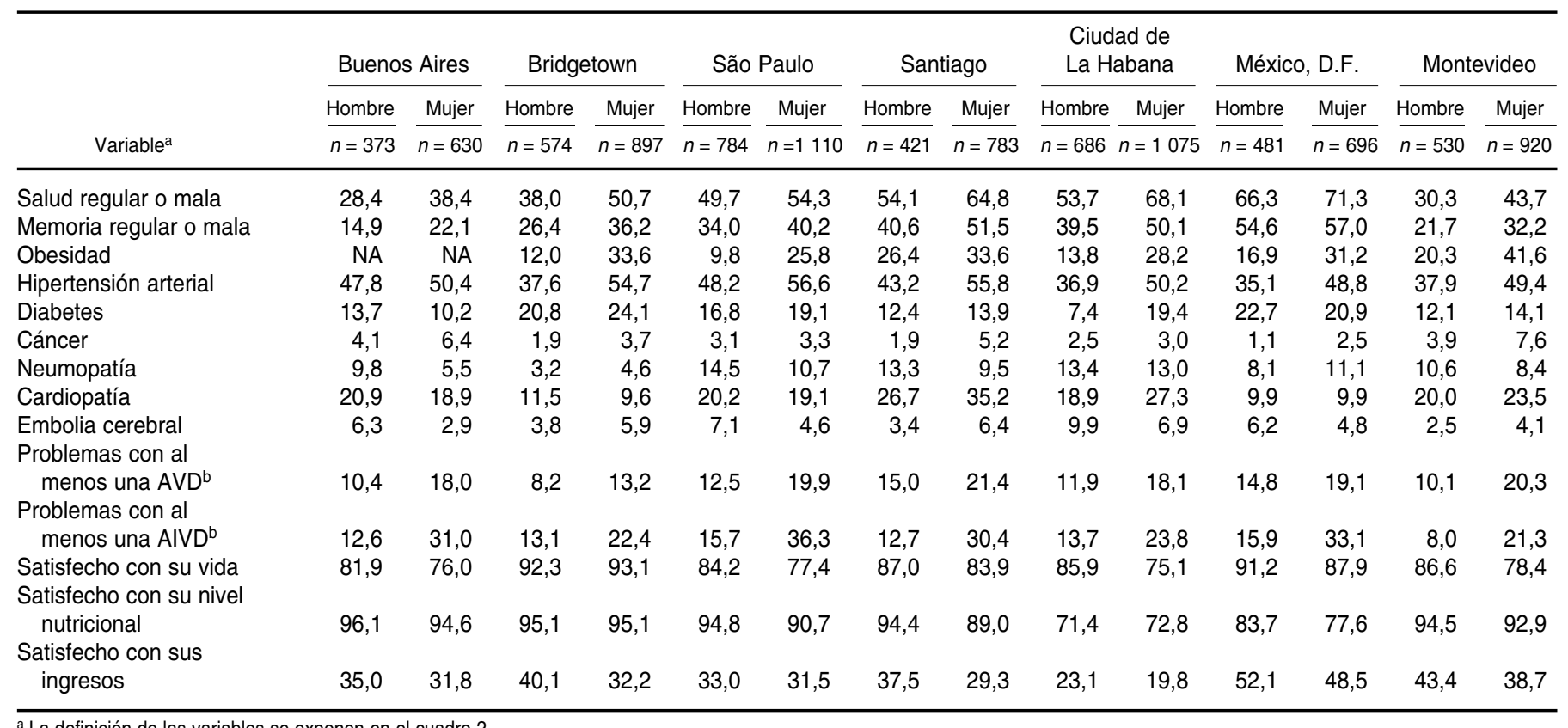

a La definición de las variables se exponen en el cuadro 2.

${ }^{b}$ AVD: actividad de la vida diaria; AIVD: actividad instrumental de la vida diaria.

zaron los siguientes indicadores de salud y bienestar como covariables del ASG: autoinforme de enfermedades crónicas (hipertensión arterial, diabetes, cáncer, neumopatía, cardiopatía y enfermedad cerebrovascular), evaluación de la propia memoria, satisfacción con su vida, con su estado nutricional y con los ingresos con que cuenta para vivir. ${ }^{6}$ Como indicadores de funcionalidad se emplearon: las dificultades con una o más actividades de la vida diaria (AVD) y con una o más actividades instrumentales de la vida diaria (AIVD). Como indicadores sociodemográficos se emplearon los datos de edad, sexo, estado civil y educación (baja: de 0 a 3 años; mediana: de 4 a 6 años; alta: 7 ó más años de estu-

\footnotetext{
6 Las preguntas se referían a si se sentía satisfecho con su vida en las últimas dos semanas (sí/no); si se consideraba bien nutrido (sí/no); si consideraba que tenía el dinero suficiente para sus gastos diarios (sí/no). La pregunta sobre la memoria se refería a si consideraba su memoria excelente, muy buena, buena, regular o mala. De manera similar al autoinforme de salud, se consideró con "mala memoria" a todo el que respondió "regular" o "mala."
}

dio), mientras que el nivel económico del hogar de residencia se evaluó mediante los servicios básicos disponibles en la vivienda y los bienes de consumo duraderos presentes en el hogar. En el cuadro 1 se presentan la lista de variables y los códigos correspondientes empleados.

Para determinar las covariables en los diferentes países estudiados se empleó la regresión con múltiples variables con datos individuales. En los modelos de regresión (probit) se utilizó el autoinforme de mala salud como variable dependiente. El efecto marginal de cada variable explicativa categórica se empleó para indicar la diferencia estimada entre la probabilidad de informar mala salud de las personas que tenían y que no tenían una característica dada, representada por la variable explicativa correspondiente, por ejemplo una enfermedad crónica. Como el valor medio de la variable dependiente (la propensión a informar mala salud) varió entre los centros urbanos participantes en el estudio SABE, se usó el efecto marginal expresado como porcentaje de dicha media, es decir, la proporción de personas que informaron tener mala salud en esa ciudad. Esto permitió comparar la importancia relativa de las covariables del ASG de las siete poblaciones. La significación estadística de los efectos marginales se calculó mediante la prueba de la $t$ de Student, con un nivel de significación de 0,10.

\section{RESULTADOS}

\section{Autoinforme de salud}

En todas las ciudades estudiadas fue muy baja la propensión a informar salud "excelente" (6\% o menos). La proporción de la población que informó tener "mala" salud fue de 5-7\% en Buenos Aires, Montevideo y Bridgetown, seguidas por Ciudad de La Habana (13\%), Santiago (18\%) y México, D.F. (19\%) (cuadro 2).

Por su parte, la distribución de los autoinformes de salud por categoría mostró que la población que informó tener una salud "excelente", "muy 
CUADRO 2. Definición de las variables de estudio. Encuesta Salud, Bienestar y Envejecimiento (SABE), 1999-2000

\begin{tabular}{|c|c|}
\hline Variable & Definición \\
\hline Autoinforme de la memoria & $1=$ regular o mala; $0=$ excelente, muy buena, o buena \\
\hline Obesidad $^{a}$ & 1 = sí (índice masa corporal $\geq 30$ ); $0=$ no \\
\hline Hipertensión arterial ${ }^{b}$ & 1 = sí; 0 = no \\
\hline Diabetes $^{b}$ & 1 = sí; 0 = no \\
\hline Cáncer ${ }^{b}$ & 1 = sí; 0 = no \\
\hline Neumopatía ${ }^{b}$ & 1 = sí; 0 = no \\
\hline Cardiopatía $^{b}$ & $1=$ sí; 0 = no \\
\hline Embolia cerebral $^{b}$ & 1 = sí; 0 = no \\
\hline Satisfecho con su vida & 1 = sí; 0 = no \\
\hline Satisfecho con su estado nutricional & $1=$ sí; 0 = no \\
\hline Satisfecho con sus ingresos & 1 = sí; 0 = no \\
\hline \multirow[t]{2}{*}{$\begin{array}{l}\text { Problemas con actividades de la vida } \\
\text { diaria (AVD) }\end{array}$} & $\begin{array}{l}1 \text { = sí; informa tener dificultad con alguna de las } \\
\text { siguientes AVD: caminar en un cuarto, vestirse, } \\
\text { bañarse, comer, entrar y salir de la cama, usar el } \\
\text { excusado. }\end{array}$ \\
\hline & $0=$ no \\
\hline \multirow[t]{2}{*}{$\begin{array}{l}\text { Problemas con actividades } \\
\text { instrumentales de la vida diaria (AIVD) }\end{array}$} & $\begin{array}{l}1 \text { = sí; informa tener dificultad con alguna de las } \\
\text { siguientes AIVD: preparar una comida caliente, } \\
\text { controlar dinero, ir solo a lugares, hacer compras de } \\
\text { alimentos, llamadas telefónicas, quehaceres ligeros } \\
\text { del hogar, quehaceres pesados del hogar, tomar sus } \\
\text { medicinas. }\end{array}$ \\
\hline & $0=$ no \\
\hline Edad & $\begin{array}{l}\text { Variables dicotómicas para las categorías: } 60-64 \text {, } \\
65-69,70-74,75-79,80 \text { o más. }\end{array}$ \\
\hline Educación & $\begin{array}{l}\text { Variables dicotómicas para niveles: bajo ( } 0 \text { a } 3 \text { años), } \\
\text { mediano ( } 4 \text { a } 6 \text { ), alto ( } 7 \text { o más). }\end{array}$ \\
\hline Servicios en la vivienda & $\begin{array}{l}\text { Número de servicios disponibles de los siguientes: } \\
\text { agua, drenaje, excusado. }\end{array}$ \\
\hline Bienes durables en el hogar & $\begin{array}{l}\text { Número de bienes disponibles de los siguientes: } \\
\text { refrigerador, lavadora, calentador de agua, televisión, } \\
\text { radio, teléfono. }\end{array}$ \\
\hline
\end{tabular}

\footnotetext{
a Los indicadores de peso y estatura no están disponibles para Buenos Aires.
}

${ }^{\text {b }} \mathrm{Si}$ alguna vez le ha dicho un doctor o enfermera que tiene la enfermedad.
$(2,6,10,18,19)$ (figura 2). Los resultados del análisis con múltiples variables de la relación entre el ASG mala o regular y los indicadores de satisfacción demostraron: 1) la importancia relativa de cada uno de estos indicadores de salud como covariable del ASG, ya que se observó el efecto de las enfermedades crónicas en los indicadores de bienestar y de funcionalidad empleados en el modelo; 2) la asociación entre las características sociodemográficas y el ASG; y 3) las diferencias o similitudes encontradas entre los siete centros urbanos con respecto a los indicadores estudiados. En el cuadro 3 se presentan los efectos marginales de un modelo probit, representados como porcentajes del valor medio de la variable dependiente en cada muestra. ${ }^{7}$

\section{Los indicadores de salud y el ASG}

Los resultados mostraron que independientemente de los elementos que formaron parte del modelo, la evaluación de la propia memoria fue el factor más fuertemente asociado con el resultado del ASG, ya que los efectos fueron de mayor magnitud para esta variable que para el resto. Las personas que informaron tener mala memoria tendieron a considerar mala su salud. Se encontró que otros indicadores de salud también estuvieron asociados significativamente con el resultado del ASG, como la satisfacción con el estado nutricional $(P<0,10$ en las siete ciudades), la satisfacción con los ingresos y con la vida ( $P<0,10$ en cinco de las ciudades), aunque con efectos marginales menores que los obtenidos para la memoria. En resumen, los resultados indiy São Paulo (alrededor de 50\%) y de Ciudad de La Habana, Santiago y México, D.F. (entre 30-40\%).

En cuanto a las tasas de ASG regular o mala según el sexo, se observó que el porcentaje de la población que informó tener salud regular o mala fue más alto en las mujeres que en los hombres en todos los centros urbanos estudiados, con excepción de São Paulo. Este es uno de los patrones más frecuentemente encontrados en investigaciones realizadas en otros países

\footnotetext{
7 También se estimaron modelos con especificaciones diferentes y los resultados no variaron. Por ejemplo, se probaron especificaciones con la suma del número de enfermedades crónicas, de AVD y de AIVD, con al menos una de las enfermedades crónicas consideradas y con arreglos residenciales. Además, cuando del modelo se eliminó la variable "evaluación de la propia memoria" no cambió el efecto de las otras variables de salud y funcionalidad, excepto el efecto de la educación, que aumentó. Por lo tanto, solamente se presentan los resultados del modelo con mejor poder pronóstico y con mayor significación para las variables de interés.
} 
FIGURA 1. Distribución de autoinformes de salud por ciudades. Encuesta Salud, Bienestar y Envejecimiento (SABE), 1999-2000

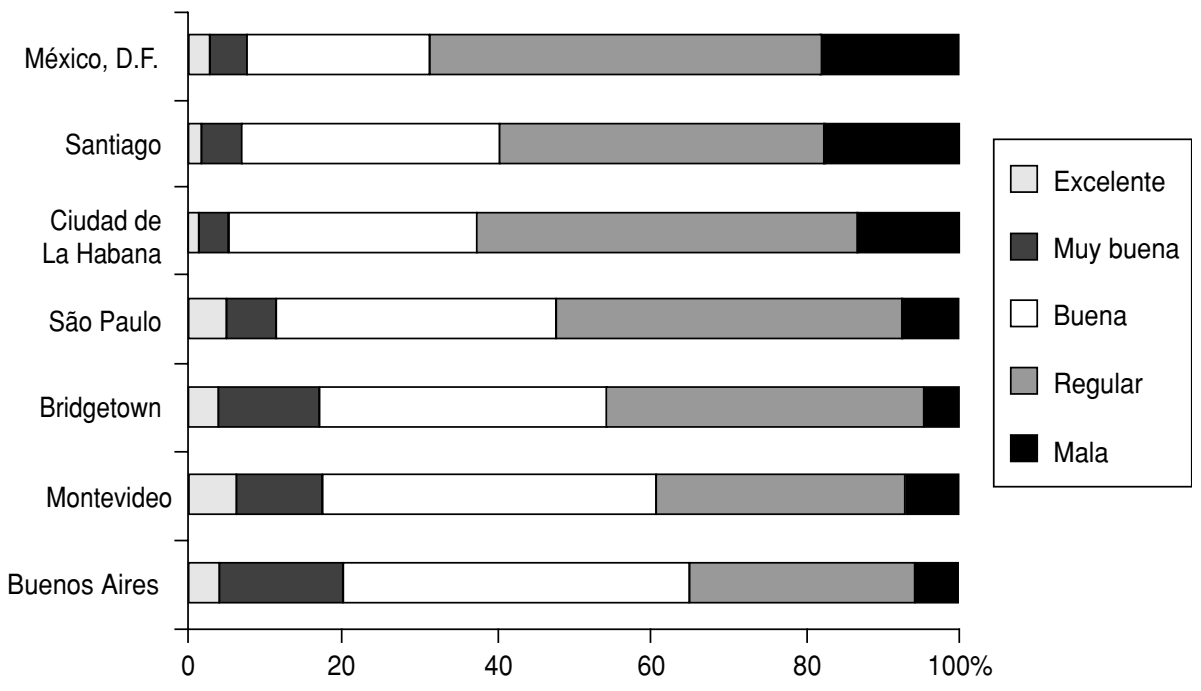

FIGURA 2. Distribución del informe de salud regular o mala por sexo y ciudad. Encuesta Salud, Bienestar y Envejecimiento (SABE), 1999-2000

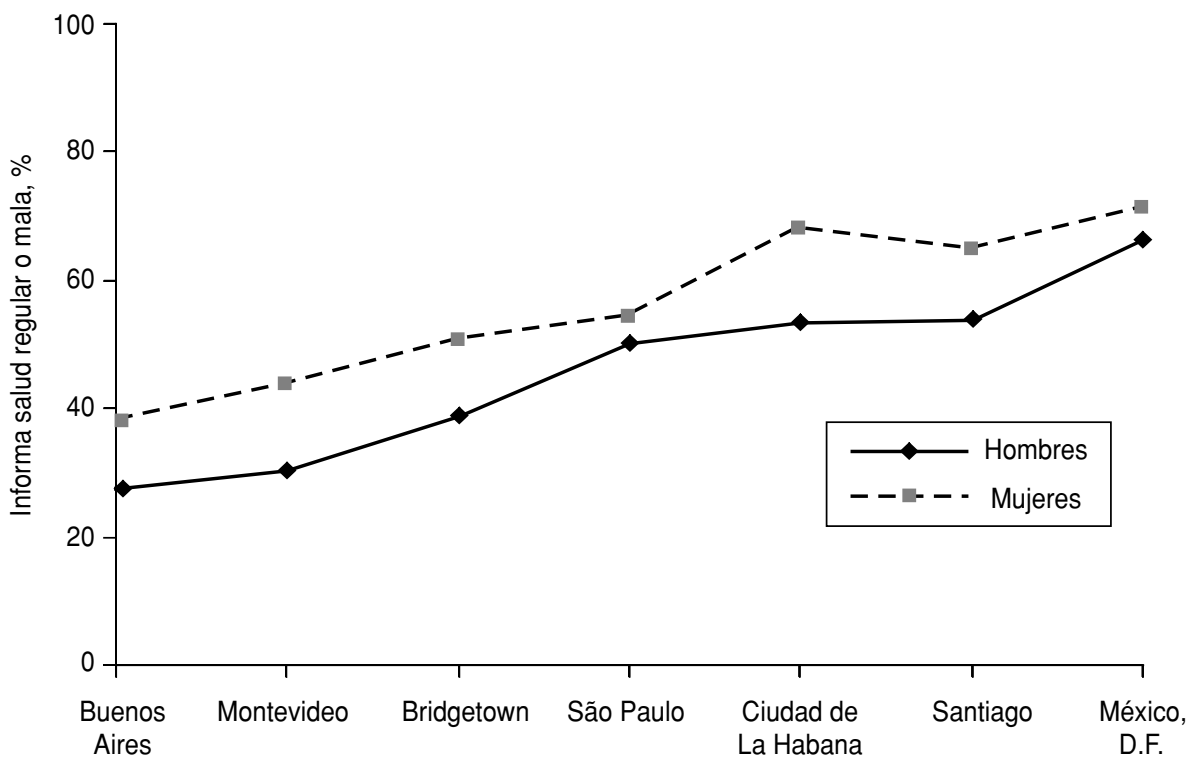

can que las variables relacionadas con la salud y el bienestar son covariables importantes del ASG en los siete centros urbanos estudiados.

Con respecto a las enfermedades crónicas o las condiciones físicas de salud, se encontró que la neumopatía y la cardiopatía tuvieron una estrecha asociación con el resultado del ASG, aunque su importancia no fue la misma en todas las ciudades estudiadas (cuadro 3). En general, estas enfermedades tuvieron mayores efectos marginales que otras enfermedades crónicas. En cuanto a las variables de funcionalidad física, el autoinforme de problemas de funcionalidad estuvo asociado con el autoinforme de mala

salud y, como se esperaba, el efecto de tener dificultades con alguna AVD fue mayor que el de tener dificultades con alguna AIVD. En resumen, la evaluación de los indicadores relacionados con el propio bienestar - ya sea la memoria, la satisfacción con la nutrición o con la vida - resultó más importante en el ASG que el padecimiento de una enfermedad crónica (cuadro 3).

\section{Los factores sociodemográficos y el ASG}

Los resultados del análisis con múltiples variables indicaron que no existían diferencias significativas entre los ASG de personas con diferentes características sociodemográficas después de ajustar según los diversos indicadores de salud y bienestar. Esto implica que las diferencias observadas entre la salud informada por hombres y por mujeres en el análisis con dos variables prácticamente desaparecieron cuando se tomaron en cuenta las enfermedades crónicas o los indicadores de bienestar o de funcionalidad. La edad, el sexo, el estado civil, las condiciones de la vivienda o los bienes de consumo duraderos en el hogar no tuvieron un efecto significativo $(P<0,10)$ en el ASG. La única variable sociodemográfica asociada con el ASG fue el nivel de educación, de manera que las personas con mayor nivel de educación presentaron menor probabilidad de informar mala salud que las personas con bajo nivel de educación, y la magnitud de este efecto relativo varió de $16 \%$ en Ciudad de La Habana hasta $46 \%$ en Buenos Aires. ${ }^{8}$

\section{Diferencias entre los países}

Aunque se encontraron similitudes entre las siete ciudades, también se encontraron algunas diferencias nota-

8 Los efectos marginales son 0,10 en Ciudad de La Habana y 0,16 en Buenos Aires, equivalentes a $16 \%$ y $47 \%$, respectivamente, si el efecto marginal se expresa como porcentaje del valor medio de la variable dependiente, es decir, la proporción que informa tener salud regular o mala $(0,10 / 0,62$ y $0,16 / 0,34$, respectivamente). 
CUADRO 3. Cambio porcentual en la probabilidad media de informar salud regular o mala. Encuesta Salud, Bienestar y Envejecimiento (SABE), 1999-2000,

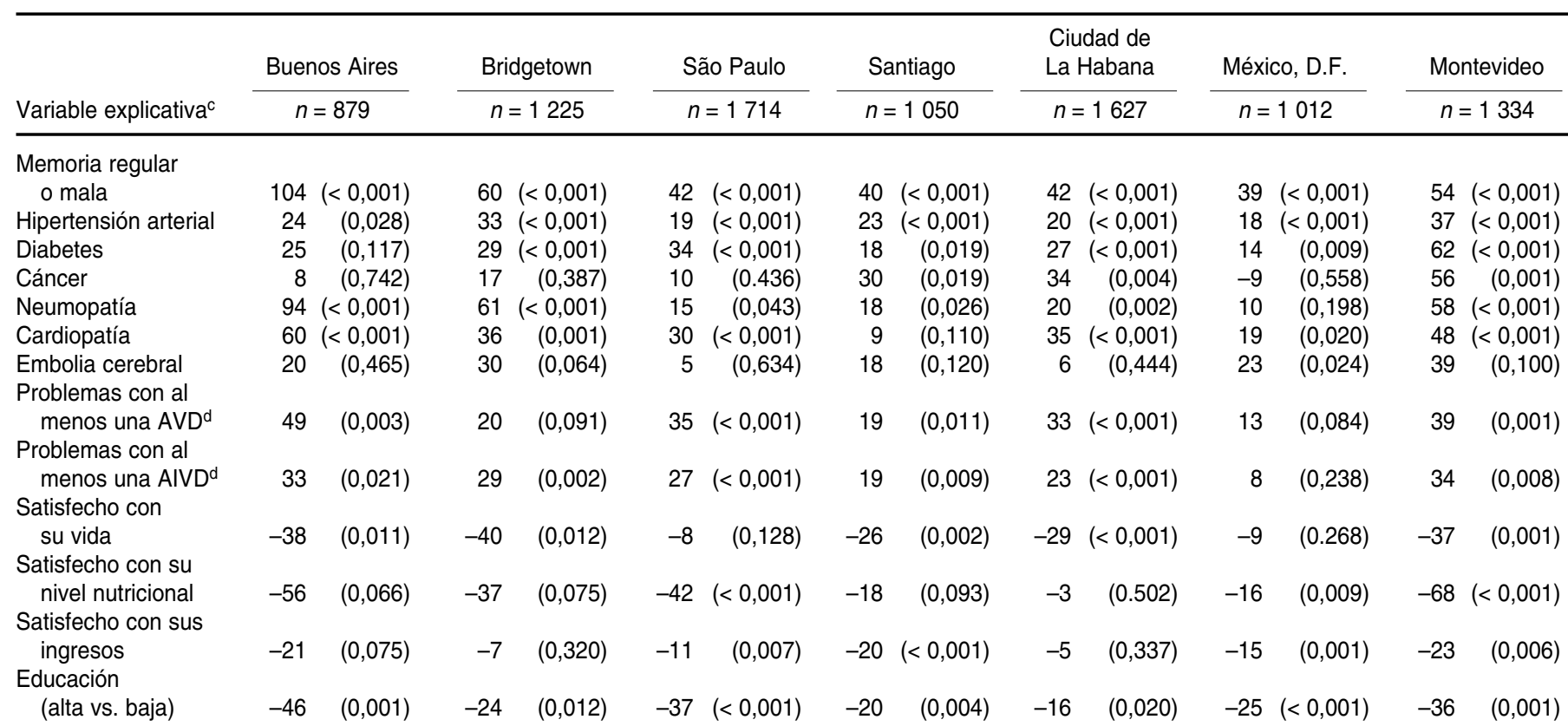

Otros controles en el modelo probit: edad, sexo, estado civil, educación, condiciones de la vivienda, bienes de consumo duraderos en el hogar.

$\begin{array}{llllllll}P \text { observada } & 0,34 & 0,48 & 0,53 & 0,63 & 0,62 & 0,69\end{array}$

Fuente: Encuesta Salud, Bienestar y Envejecimiento (SABE).

${ }^{a}$ El cuadro presenta los efectos marginales de un modelo de regresión probit, expresados como porcentaje del valor medio de la variable dependiente, es decir, como la proporción que informa tener salud regular o mala en cada ciudad.

${ }^{b}$ En paréntesis se presentan los valores de $P$. Nivel de significación de 0,05.

${ }^{c}$ La definición de las variables se encuentran en el cuadro 2.

${ }^{\mathrm{d}}$ AVD: actividad de la vida diaria; AIVD: actividad instrumental de la vida diaria.

bles. Montevideo fue una de las ciudades con mayor número de autoinformes de mejor salud general y la única ciudad donde la evaluación de la propia memoria no fue la covariable más fuertemente relacionada con el ASG (con un cambio porcentual estimado de $54 \%$ ), pues fue mucho más importante la satisfacción nutricional (con un cambio de 68\%). De hecho, la evaluación de la memoria contribuyó relativamente menos (octavo factor en importancia), en comparación con los demás indicadores de salud del modelo. Otra excepción fue que la neumopatía crónica tuvo efectos relativamente más altos sobre el ASG en Buenos Aires, Bridgetown y Montevideo, las ciudades con mejor salud general. Por otro lado, es notable la ausencia de enfermedades cardiovasculares entre las covariables principales asociadas con la salud general en Santiago.
En México, D.F., donde la población manifestó una elevada tendencia a percibir su salud como mala, el modelo con múltiples variables mostró excepciones notables. Por ejemplo, solamente en México, D.F. se encontró que la neumopatía crónica y los problemas para realizar AIVD no fueron covariables significativas $(P>0,10)$ del ASG. $Y$, sin embargo, México, D.F. fue la única ciudad donde la enfermedad cerebrovascular fue una covariable significativa $(\mathrm{P}<0,05)$, segunda en orden por su importancia relativa, superada solamente por la evaluación de la propia memoria.

Otro dato interesante fue que los efectos marginales resultaron relativamente mayores en Buenos Aires, Bridgetown y Montevideo que en las ciudades con menos autoinformes de buena salud. En México, D.F., donde se encontró el mayor número de autoinformes de mala salud, los efectos marginales de todas las variables fueron menores que en el resto de las ciudades. Por ejemplo, el cambio porcentual en la probabilidad media de informar mala salud general asociada con la mala memoria en México, D.F. fue de $39 \%$, mucho menor que en Buenos Aires (104\%) y en Bridgetown $(60 \%)$. Este resultado indica que en las ciudades donde la salud es mejor, el ASG tiende a estar relacionado en mayor medida con la salud física, el bienestar o la funcionalidad.

En Bridgetown se encontraron diferencias entre los ASG de todos los grupos de edad. El efecto marginal fue positivo, lo que indica que a mayor edad había mayor probabilidad de percibir la salud como mala, excepto en el grupo de 80 años o más. Solamente se encontraron diferencias por edad en las personas de 80 años o más de México, D.F. y Santiago, pero el efecto fue 
FIGURA 3. Probabilidad de informar salud regular o mala en personas que se ajustan al prototipo 1 en las ciudades estudiadas ${ }^{\mathrm{a}}$

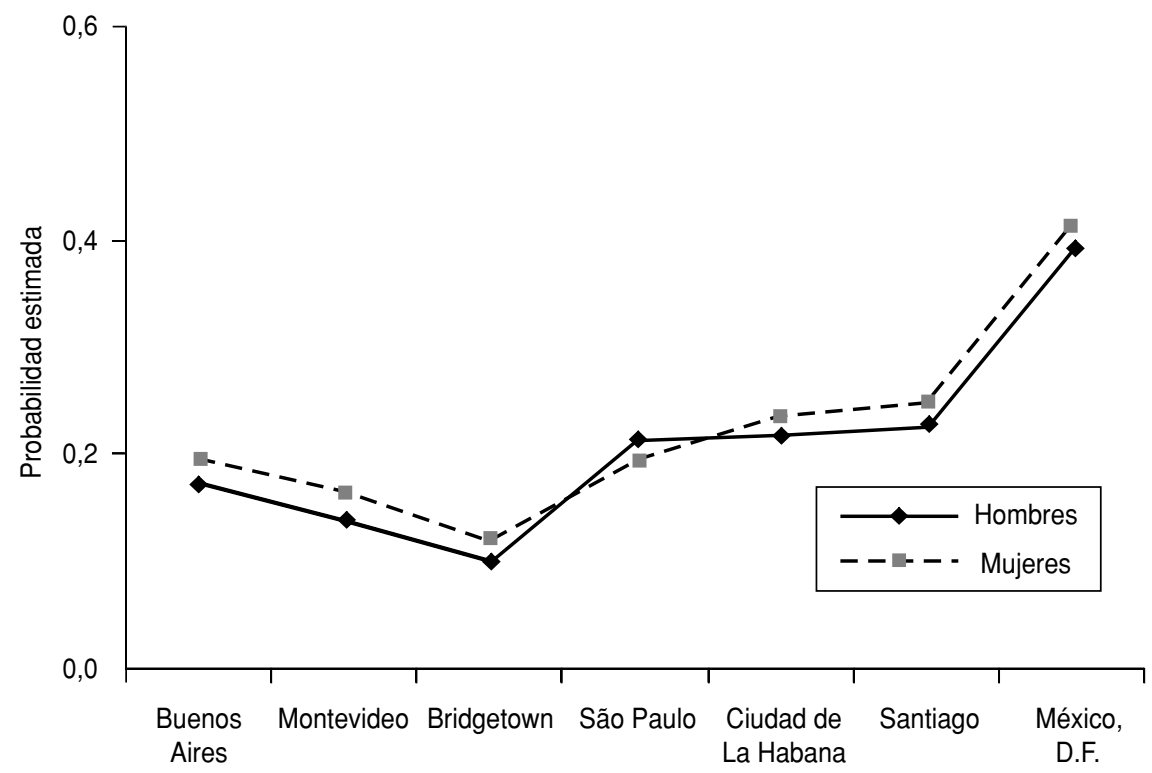

Fuente: Encuesta Salud, Bienestar y Envejecimiento (SABE), 1999-2000.

a Prototipo 1: hombres o mujeres de 60 a 64 años de edad, casados, sin problemas para realizar AVD o AIVD y sin enfermedades crónicas.

en sentido inverso al encontrado en Bridgetown. Es decir, que en estos centros urbanos las personas de 80 años de edad o más informan tener mejor salud que las de menor edad $(P<0,10)$.

Para ilustrar las diferencias en cuanto a la evaluación de la propia salud en los centros urbanos estudiados, se calculó la probabilidad de informar mala salud mediante el modelo con múltiples variables para dos prototipos de personas con características iguales en las siete poblaciones. En la figura 3 se presenta la probabilidad de informar mala salud de una persona media de 60 a 64 años de edad, casada, que no padeciera de las enfermedades crónicas utilizadas en el modelo y que no tuviera dificultades para realizar AVD o AIVD. Los resultados revelaron que aun cuando se consideran personas con las mismas características sociodemográficas y de salud (sanas), hay una mayor propensión a evaluar su salud como mala en México, D.F. $(0,41)$ que en Ciudad de La Habana o Santiago $(0,23)$ y que en Bridgetown $(0,11)$. Además, esta propensión fue muy similar en hombres y mujeres dentro de cada una de las ciudades es- tudiadas, lo que significa que las diferencias en la evaluación general de la propia salud en estas poblaciones se mantuvieron aun cuando se ajustó según diversos indicadores de salud y aspectos sociodemográficos. La probabilidad de que un viudo o viuda de 70 a 74 años de edad con dificultades para realizar al menos una AVD y una AIVD considere su salud como regular o mala fue mayor en Ciudad de La Habana, México, D.F. y Santiago (alrededor de 0,6$)$ que en Montevideo $(0,2)$ o Buenos Aires $(0,3)$ (figura 4). Aunque las diferencias por sexo se redujeron al controlar según las condiciones de salud, los resultados indicaron que en Buenos Aires y Ciudad de La Habana, las mujeres con estas características tienden a percibir peor su salud que los hombres.

\section{DISCUSIÓN}

En este artículo se valora el ASG como indicador de salud y se analizan sus covariables - especialmente aquellas cuya evaluación mediante el au- toinforme puede considerarse subjetiva - en muestras representativas de la población de 60 años de edad o más que residía en hogares privados en siete centros urbanos de América Latina y el Caribe. Los datos del estudio multicéntrico SABE se obtuvieron mediante encuestas y protocolos diseñados para comparar las poblaciones en estudio.

El ASG es uno de los indicadores más usados para comparar la salud de los adultos mayores de diferentes países debido a que es un indicador fácil de obtener y a que refleja múltiples aspectos de la salud que pueden resultar difíciles de captar por otros métodos en muestras representativas de la población.

En la mayoría de los países, la evaluación de la propia salud estuvo fuertemente asociada con la evaluación de la propia memoria, seguida del padecimiento de enfermedades crónicas, como la neumopatía, cardiopatía, y en algunos casos la diabetes. Otro elemento asociado con el ASG fue la satisfacción con el nivel nutricional. En general, estos factores estuvieron más asociados con la salud general que los problemas de funcionalidad. Este resultado puede indicar que en el universo estudiado, los problemas de la memoria, de la nutrición y el padecimiento de enfermedades crónicas son más frecuentes en la población de 60 años de edad o más, y por lo tanto son mejores indicadores de mala salud que la pérdida de funciones que afectan solamente a los adultos de mayor edad. Es necesario resaltar que este hallazgo contradice lo encontrado en estudios realizados en Asia, donde los problemas funcionales estuvieron más fuertemente asociados con la salud en general que las enfermedades físicas o mentales (18-19). Estos resultados confirman la idea de que el ASG en América Latina presenta sus particularidades, posiblemente debido a la etapa de transición epidemiológica en que se encuentra la Región actualmente. Sin embargo, esta hipótesis requiere de investigaciones más detalladas.

Cuando los resultados obtenidos se ajustan según las enfermedades y la satisfacción, desaparecen las diferencias en la evaluación de la propia salud por 
FIGURA 4. Probabilidad de informar salud regular o mala en personas que se ajustan al prototipo 2 en las ciudades estudiadas ${ }^{a}$

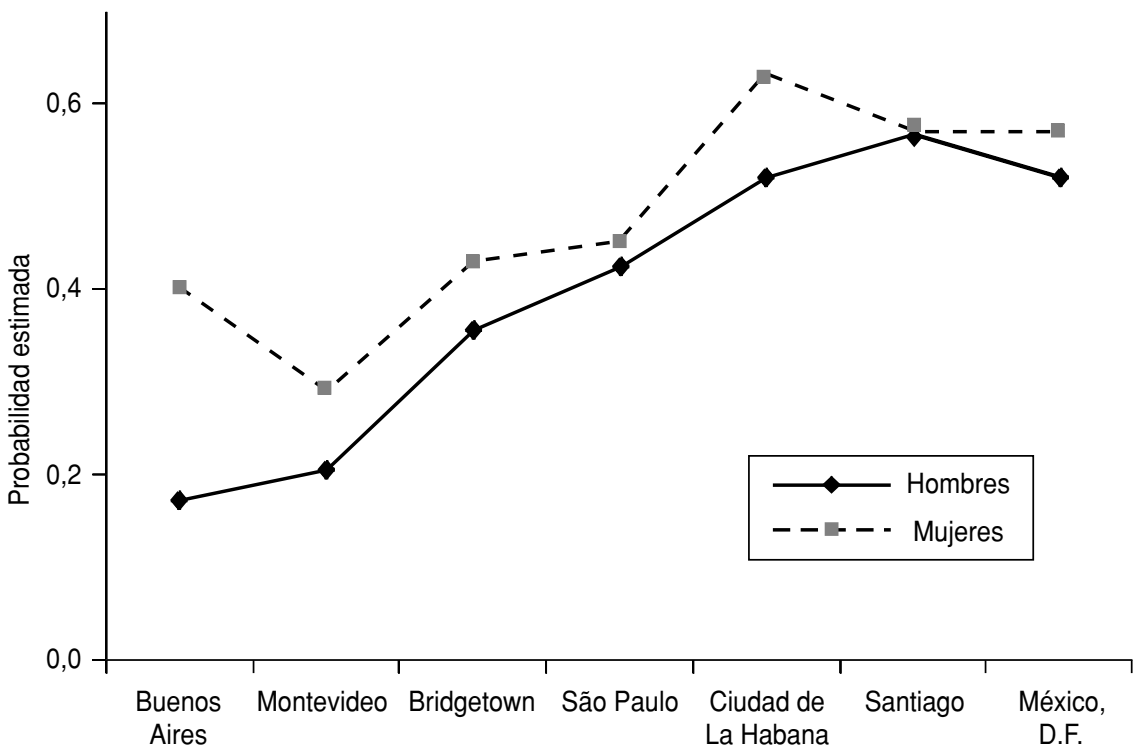

Fuente: Encuesta Salud, Bienestar y Envejecimiento (SABE), 1999-2000.

a Prototipo 2: hombres o mujeres de 70 a 74 años edad, viudos, con problemas para realizar AVD y AIVD, sin enfermedades crónicas.

personas con diversas características sociodemográficas, como la edad, el sexo, el estado civil, y los servicios y los bienes disponibles en la vivienda. Al controlar según las covariables de salud, el único factor que presentó un claro efecto independiente sobre el resultado del ASG fue el nivel educacional, y las diferencias se encontraron solamente entre las personas con el mayor nivel de educación respecto a las que tienen bajo nivel. Las diferencias entre el ASG de hombres y mujeres se deben a desigualdades en el estado de salud, bienestar y funcionalidad y no a la mayor o menor propensión de uno $u$ otro sexo a informar un estado de salud determinado. Este resultado coincide con lo observado en algunos países de Asia, donde una vez controladas según los problemas de salud física y mental, las diferencias por factores sociodemográficos desaparecen, excepto por el nivel educacional (19).

Los factores sociodemográficos asociados con el ASG dependen de diversas facetas de la salud. En América Latina y el Caribe, las mujeres generalmente informaron gozar de peor salud general que los hombres, posible- mente debido a que las mujeres tienen más problemas de salud y de bienestar que los hombres. Sin embargo, las personas con mayor educación informaron gozar de mejor salud general que las de menor nivel de educación, aun con condiciones de salud similares.

La percepción de gozar de mejor salud en el grupo de 80 años o más con respecto a grupos de adultos mayores más jóvenes en México, D.F. y Santiago coincide con lo encontrado por otros autores que informaron que mediante un modelo con múltiples variables, la percepción de buena salud fue mayor en personas de edad avanzada que en otras más jóvenes con estado de salud similar. Esto puede deberse a que los de mayor edad tienen un grupo de referencia menos saludable (20). Otra posible explicación es que las personas de edad avanzada son más optimistas con respecto a su salud a medida que envejecen, porque se han acostumbrado y perciben las enfermedades de manera más ligera u optimista que las más jóvenes; o que las personas de 80 años o más de México, D.F. y Santiago que vivieron momentos históricos difíciles pueden sentirse más optimistas que otros grupos de edad, aunque este efecto no necesariamente se mantendrá en el futuro. Por último, esto puede ser resultado del efecto de la supervivencia, es decir, los que llegan a los 80 años de edad constituyen el grupo de mayor optimismo, mientras que los más pesimistas con respecto a su salud pueden haber fallecido ya (2, 21). El análisis de este tema con mayor detalle y datos longitudinales puede contribuir a encontrar la explicación de este resultado.

Los factores que influyen en la evaluación de la propia salud difieren de un país a otro y esta diversidad de patrones puede deberse a la etapa de transición epidemiológica en que se encuentra una población dada. Por ejemplo, en poblaciones más saludables, la percepción de gozar de mejor salud puede depender en mayor medida de la salud emocional, de las enfermedades crónicas o de los problemas de funcionalidad, mientras que en poblaciones con peor salud, la evaluación general de la propia salud puede estar más afectada por otros problemas de salud que no se han tenido en cuenta en este modelo, como las enfermedades infecciosas. Otra posible explicación de las diferencias encontradas es que personas con niveles similares de salud perciban de manera diferente su estado de salud en dependencia de determinados elementos estructurales de los sistemas sanitarios nacionales - como la calidad de los servicios- o de la importancia que se les da a las enfermedades que sufren. Sin embargo, la tendencia a informar un peor estado de salud en Ciudad de La Habana, Santiago y México, D.F., aun después de ajustar según otros indicadores de salud y factores sociodemográficos, puede deberse también a otros factores no identificados en este estudio. Se requieren nuevas investigaciones para poder explicar las diferencias que persisten entre las siete ciudades.

Una de las ventajas del estudio $\mathrm{SABE}$ es la posibilidad de comparar los siete centros urbanos estudiados, ya que se realizó de forma coordinada con protocolos y diseños similares en los siete países. Su principal limitación reside en que las observaciones abar- 
can solamente a la población de adultos mayores de esas ciudades y no toma en cuenta la población rural y de otras zonas urbanas. Además, no se tuvo en cuenta a las personas de 60 años o más que vivían en arreglos institucionales o viviendas colectivas, como hospitales, residencias geriátricas, casas de huéspedes, hospicios, asilos y similares. Esta es una característica que se debe tener en consideración al comparar los resultados obtenidos en los diferentes países, ya que la tasa de institucionalización de este segmento de la población puede variar significativamente de una ciudad a otra. ${ }^{9}$ Por ello, aunque a lo largo del

\footnotetext{
9 Aunque no existen censos específicos de la población de adultos mayores que viven en viviendas colectivas o instituciones, la propensión a vivir en instituciones de cuidado para adultos se considera en general baja en la mayor parte de los países de la Región. Según informantes claves entrevistados, Montevideo y Bridgetown son las ciudades con mayor oferta y demanda de este tipo de residencia o instituciones.
}

trabajo se compararon los niveles de algunas variables entre los centros urbanos estudiados, se hizo mayor hincapié en comparar las asociaciones entre los factores o las variables.

El presente trabajo multinacional demostró la utilidad del ASG para conocer el estado de salud de los adultos mayores en América Latina y el Caribe. Estos resultados indican que las personas de 60 años de edad o más que residían en la comunidad en los siete centros urbanos estudiados reflejaron en sus autoinformes diversas facetas de su salud, entre ellas el padecimiento de enfermedades crónicas, su grado de satisfacción con el nivel de nutrición y con la vida, su percepción del estado de la propia memoria y los problemas de funcionalidad que sufrían. El estado de la memoria influyó más en la evaluación general de la propia salud que otros elementos de salud y de bienestar, aunque el método de análisis empleado no permitió estable- cer relaciones causales. Se deben emprender estudios más detallados que permitan establecer el papel que desempeña la salud emocional en la demanda de atención sanitaria de los adultos mayores en América Latina y el Caribe y determinar si existe asociación entre el resultado del ASG y el uso de los servicios de salud.

Agradecimientos. Este trabajo se realizó parcialmente bajo el patrocinio de la Organización Panamericana de la Salud como parte de un acuerdo con el Instituto Nacional de Envejecimiento de los Institutos Nacionales de Salud de los Estados Unidos de América. Los autores agradecen la ayuda de Lekha Subaiya en el análisis de los datos, así como los comentarios a una versión anterior de Juan José Díaz, ambos del Maryland Population Research Center, Maryland, EUA.

\section{REFERENCIAS}

1. Power C, Matthews S. Origins of health inequalities in a national population sample. Lancet. 1997;350:1584-9.

2. Idler EL, Benyamini Y. Self-rated health and mortality: a review of twenty-seven community studies. J Health Soc Behav. 1997;38(1): 21-37.

3. Power C, Manor O, Matthews S. The duration and timing of exposure: effects of socioeconomic environment on adult health. Am J Public Health. 1999;89:1059-65.

4. Smith J. Healthy bodies and thicker wallets: the dual relation between health and socioeconomic status. J Econ Perspect. 1999;13(2): $145-66$.

5. Hurd M, Kapteyn A. Health, wealth, and the role of institutions. J Hum Resour. 2003;38(2): 386-415.

6. Wong R. La relación entre salud y nivel sociodemográfico entre adultos mayores: diferencias por género. En: Salgado de Snyder V, Wong R, eds. Envejeciendo en la pobreza: género, salud y calidad de vida. México, D.F.: Instituto Nacional de Salud Pública; 2004. Pp. 97-122.

7. Angel R, Gronfein W. The use of subjective information in statistical models. Am Sociol Rev. 1988;53:464-73.

8. Wallace R, Herzog AR. Overview of the health measures in the Health and Retirement Study. J Hum Resour. 1995;30(Suppl):S84-107.
9. Baker M, Stabile M, Deri C. What do selfreported, objective, measures of health measure? J Hum Resour. 2004;39(4):1067-93.

10. Hermalin AI, ed. The well-being of the elderly in Asia: a four-country comparative study. Ann Arbor: University of Michigan Press; 2002

11. Palloni A, Pinto-Aguirre G, Peláez M. Demographic and health conditions of ageing in Latin America and the Caribbean. Int J Epidemiol. 2002;31:762-71.

12. Albala C, Lebrão ML, León Díaz EM, HamChande R, Hennis AJ, Palloni A, et al. Encuesta Salud, Bienestar y Envejecimiento (SABE): metodología de la encuesta y perfil de la población estudiada. Rev Panam Salud Publica. 2005;17(5-6):307-22.

13. Markides KS, Lee DJ. Predictors of well-being and functioning in older Mexican-Americans and Anglos: an eight-year follow-up. J Gerontol Soc Sci. 1990;45(1):569-73.

14. Krause NM, Jay GM. What do global selfrated health items measure? Med Care. 1994; 32(9):930-42.

15. Idler EL, Kasl SV. Self-ratings of health: do they also predict change in functional ability? J Gerontol Soc Sci. 1995;50B(6):S344-53.

16. Reuben DB, Siu AL, Kimpau S. The predictive validity of self-report and performance-based measures of function and health. J Gerontol Med Sci. 1992;47(4):M106-10.
17. Fylkesnes K, Forde OH. The Tromso Study: predictors of self-evaluated health-has society adopted the expanded health concept? Soc Sci Med. 1991;32(2):141-6.

18. Rahman MO, Barsky AJ. Self-reported health among older Bangladeshis: how good a health indicator is it? Gerontologist. 2003;43(6): 856-63.

19. Zimmer Z, Natividad J, Ofstedal, MB, Lin H. Physical and mental health of the elderly. En: Hermalin AI, ed. The well-being of the elderly in Asia: a four-country comparative study. Ann Arbor: University of Michigan Press; 2002.

20. Cockerham WC, Sharp K, Wilcox JA. Aging and perceived health status. J Gerontol. 1983; 38(3):349-55.

21. Idler EL. Age differences in self-assessments of health: age changes, cohort differences, or survivorship? J Gerontol Soc Sci. 1993;548(6): S289-300.

Manuscrito recibido el 25 de febrero de 2005. Aceptado para publicación, tras revisión, el 29 de marzo de 2005. 
ABSTRACT Objectives. To evaluate self-reported general health (SRGH) as a health indicator and to analyze its covariates in people 60 years old or older living in private homes in seven cities of Latin America and the Caribbean.

\section{Self-reported general health in older adults in Latin America and the Caribbean: usefulness of the indicator}

Methods. This cross-sectional descriptive study was based on data from the Health, Well-Being, and Aging survey (Salud, Bienestar y Envejecimiento, or "SABE survey"), which was carried out in 1999 and 2000 in Bridgetown, Barbados; Buenos Aires, Argentina; Havana, Cuba; Mexico City, Mexico; Montevideo, Uruguay; Santiago, Chile; and São Paulo, Brazil. The survey looked at the demographic and socioeconomic characteristics of the participants, several health indicators (self-reported chronic diseases, depression, and cognitive features), the social and family support network, the use of health services, reported and observed functionality, the respondent's income, and the durable consumer goods in the household. In probit regression models, selfreported fair or poor health was used as the dependent variable. The marginal effect of each categorical explanatory variable was used to indicate the difference between the probability of reporting poor health by persons who did or did not have a given characteristic.

Results. In all the cities studied the self-reporting of "excellent" health was very low (6\% or less). The results of the multivariate analysis of the relationships between SRGH and covariates showed: (1) the relative importance of several health indicators as covariates of SRGH, (2) the association between sociodemographic characteristics and SRGH, and (3) the differences or similarities found among the seven cities with respect to the relationships studied. The level of self-rated good health was highest in Buenos Aires and Montevideo (60\%), followed by Bridgetown and São Paulo (around $50 \%$ ) and Havana, Santiago, and Mexico City (between 30\% and 40\%). The respondents' evaluation of their memory was the factor that was most strongly related to SRGH, followed by satisfaction with nutritional status and satisfaction with life.

Conclusions. The SRGH captured multiple facets of the health of the older adults, such as suffering from chronic diseases, the degree of satisfaction with the level of nutrition and with life, perception of memory, and any functional problems. More detailed studies should be undertaken to try to determine the role that the emotional health of older adults in Latin America and the Caribbean plays in the demand for health care, and to determine if there is some association between SRGH and the use of health services.

Keywords Aged; aged, 80 and over; activities of daily living; health status. 